\title{
Antibacterial Resistance of Escherichia coli from Rectal Swabs of Synanthropic Rodents Trapped from Household Compounds in Wolaita Zone, Southern Ethiopia
}

\author{
Asfaw Tora*, Yishak Abraham \\ College of Natural and Computational Science, Department of Biology, Wolaita Sodo University, Ethiopia \\ *Corresponding Author: Asfaw Tora, College of Natural and Computational Science, Department of \\ Biology, Wolaita Sodo University, Ethiopia
}

\begin{abstract}
:
Background: Antibacterial resistance (ABR) varies between regions and countries depending upon consumption degree of both animal and human antibiotics, which is guided and regulated by the antibiotic policies of a particular country.

Objective: This study was aimed to test antibacterial resistance of Escherichia coli from rectal swabs of synanthropic rodents in Wolaita Zone, Southern Ethiopia.

Methods: A total of 77 rodents were trapped and standard methods were used to isolate E. coli from all the rodent species comprising Stenocephalemys albipes 24(31.2\%), Mus mohamet 18(23.4\%), Arvicanthis spp 15(19.5\%), Mastomys erythroleucus 12(15\%), Gerbriliscus species 4(5.2\%), Crocidura oliveri 3 (3\%) and Acomys wilsoni 1(1.3\%). Disc diffusion method was used to determine the antimicrobial resistance pattern of the E. coli against twelve antimicrobial agents: Amoxicillin, Chloramphenicol, Ciprofloxacin, Nalidixic acid, Ampicillin, Gentamicin, Nitrofuratoin, Ceftazidime, Cloxacillin, Ceftriaxone, Tetracycline, and Amoxicillinclavunic acid.

Results: The antibiogram revealed that $31.38 \%$ of the E. coli isolates were resistant to all drugs tested except Ciprofloxacin, Gentamicin and Chloramphenicol. Complete resistance to amoxicillin and Amoxicillinclavunic acid was observed in the E.coli isolates.

Conclusion: This study demonstrated that synanthropic rodents in the household compounds may have been exposed to materials containing antibacterial residues and that rodents carry and transfer drug resistant bacteria which can pose a public health hazards to humans and other domestic animals. The need to introduce and sustain rodent control programme is implicated. Special emphasis is also needed to be given for the rational use of drugs as part of controlling antibacterial resistance by bacterial pathogens.
\end{abstract}

Keywords: Escherichia coli, antibacterial resistance, rodents.

\section{INTRODUCTION}

Rodents harbor various bacteria resistant to anti bacterials used in both humans and animals. Escherichia coli have been isolated from rodents in different parts of the world [1]. The transmission dynamics of $E$. coli among synanthropic rodents, livestock and humans may contribute for the development of antibacterial resistance [2,3,4]. Although there is controversy over the natural ecology of resistant bacterial population, these results can incriminate rodents as an important source of antibacterial resistant isolates which may infect humans and other animals [5]. Escherichia coli is an important opportunistic pathogen in some parts of the body with increasing antibacterial resistance [6].

Antibacterial resistance (ABR) could have arisen from the fact that rodents get into contact with antimicrobials through various sources in the environment, for example, food, water and sewer systems [7]. Interestingly, higher resistance ware found for Amoxacillin and Amoxacillin-clavulanic acid which are more commonly used for treatment of infection in humans and animals. This can also support the idea that ABR can occur naturally in a bacterial population not exposed to antimicrobials [8]. ABR can be transferred rapidly through a susceptible bacterial population in vitro. The possibility of transfer in the normal gut (in vivo), however, can be detected only at a very low rate [9]. 
Considering the potential public health risk posed by rodents to livestock, pet animals, and humans, the current study was conducted to test ABR of E. coli from rectal swabs of synanthropic rodents trapped from rural household compounds in Fate and Abaya Chokare kebelles of Damot Gale and Humbo districts, respectively in Wolaita Zone, Southern Ethiopia.

\section{MATERIALS AND MeTHOdS}

\subsection{Study Area and Period}

Wolaita zone is one of the 13 administrative zones in the Southern Nations Nationalities and Peoples Region (SNNPR). The zone has an area of $44471.3 \mathrm{~km}^{2}$ and located $320 \mathrm{kms}$ south of Addis Ababa, the capital of the country. The total population of the zone is estimated to be 1,527908. It is one of the most densely populated zones in Ethiopia with an average 290 people $/ \mathrm{km}^{2}$. The zone is divided into 12 administrative districts.

A total of 2 rural kebeles that are located in Humbo (geographical coordinates: 37N0382846), UTM 0729850; elevatiom: 1186 MSL) and Damot Gale (geographical coordinates: 37N0367959), UTM 0769846; elevation: 2073 MSL districts were selected purposefully for trapping of rodents based on consultation with district agricultural bureaus about the general hygiene profiles and rodent problems in the areas. In the kebeles, houses are interspaced approximately 20-50 m and are often surrounded by crop fields as well as patches of shrubs and bushes. Moreover, it was very common to observe piles of wood, crop residue and traditional crop storage structures in the household compounds in the kebeles. The household compounds generally consisted of 1 house with no separate kitchen and an animal house. A total of 10 household compounds ( 5 from Fate kebele and 5 from Abyachokare) were selected for trapping the rodents for screening of Escherichia coli. Rodents were trapped from December 2015 to March 2016.

\subsection{Study Animals}

The study animals were synanthropic rodents in Fate kebele of Damot Gale district and Abaya Chokare kebele of Humbo district in Wolaita Zone, Southern Ethiopia.

\subsection{Sample Size Determination}

The sample size of rodents trapped for rectal swab collection was determined using the prevalence of 5.3\% Escherichia coli from rodent fecal samples as reported by Mushtaq-UL-Hassan et al. [10].

The formula [11] was used for calculating the sample size.

$$
\mathrm{N}=\frac{z^{2}(p)(1-p)}{\mathrm{d}^{2}}=\frac{(1.96)^{2} \times 0.053(1-0.053)}{(0.05)^{2}}=77
$$

Where:

$\mathrm{N}$ = sample size,

$\mathrm{z}=$ desired value for level of confidence $=1.96$,

$\mathrm{d}=$ desired level of precision, 0.05 , and

$\mathrm{p}=$ reported prevalence $=0.053$.

Accordingly, 77 rodents were trapped for collection of rectal swab samples.

\subsection{Rodent Collection}

Rodents were trapped live by using Sherman LFA live traps $(7.5 \times 9.0 \times 23.0 \mathrm{~cm}$, HB Sherman trap, Tallahassee, USA) baited with peanut butter from 5 purposively selected household compounds from each kebele. In this study, 8 traps were placed in each household compound, 4 inside houses (near beds, food and clothing cabinets, holes, and hide or jute sacks) and 4 outside houses (near walls, crop storage structures and live fencings within the compound). Trapping was repeated in the selected household compounds throughout the trapping period. Trapping was conducted for one night, every month. Traps were checked in the morning and captures were labeled. Trapped animals were then transported to the Biomedical Science laboratory of the Department of Biology, Wolaita Sodo University. The animals were sacrificed by cervical dislocation and external body measurements 
(weight, lengths of the body, ear, tail and hind foot were recorded). Sex and species of the trapped animals were identified with expert guidance.

\subsection{Collection of Rectal Swabs}

Strict aseptic procedure was followed to collect fecal samples from the rectum of the rodents. Sterile saline-moistened cotton-tipped applicator sticks were used to collect rectal swabs from each of the rodent. The rectal swab from each rodent was transferred into sterile buffered peptone water in sterile screw capped test tubes and stored in $4^{\circ} \mathrm{c}$ refrigerator until culturing the target bacterium, Escherichia coli.

\subsection{Isolation of Escherichia coli}

The rectal swab from each rodent was inoculated onto MacConkey agar (Oxoid Ltd., Detroit, Michigan, USA) and Xylose-Lysine Deoxycholate Agar (Oxoid Ltd., Detroit, Michigan, USA) and incubated aerobically for $24 \mathrm{~h}$ at $37^{\circ} \mathrm{C}$.

\subsection{Preservation of Escherichia coli Isolates}

The pure culture was stored in sterile $80 \%$ glycerin and was used as stock culture. The equal volume of $80 \%$ glycerin and bacterial culture were mixed and capped tightly and stored at $-20^{\circ}$ until antimicrobial resistance test was done. The isolated organisms were given codes for convenience.

\subsection{Antimicrobial Susceptibility Test}

\subsubsection{Disc Diffusion Method}

Modified Kirby-Bauer disk diffusion method was used to test the susceptibility of Escherichia coli isolates to 12 different antimicrobial agents including Ampicillin (Amp,10 $\mu \mathrm{g}$ ), Tetracycline (Tet,30 $\mu \mathrm{g})$, Chloramphenicol (Chl, $30 \mu \mathrm{g})$, Nitrofurantione (F,100 $\mu \mathrm{g})$, Cloxacilline (Ob, $5 \mu \mathrm{g})$, Amoxicilin (Amx, $2 \mu \mathrm{g}$ ), Nalidicic acid (Na, $30 \mu \mathrm{g}$ ), Gentamycin (Gen, $30 \mu \mathrm{g}$ ), Ceftazidime (Caz, $30 \mu \mathrm{g}$ ), Ceftriaxone (Cro, $30 \mu \mathrm{g}$ ), Ciprofloxacin (Cip, $5 \mu \mathrm{g}$ ), and Amoxicillin-clavulanic acid (Amc, $30 \mu \mathrm{g}$ ) (Oxoid,UK). The inocula were prepared by growing E. coli on MacConkey agar plates and 3-5 colonies from the plate were transferred with inoculating loop into $5 \mathrm{ml}$ of Trypticase soya broth $\left(\mathrm{BBL}^{\mathrm{TM}}\right.$ Trypticase ${ }^{\mathrm{TM}}$ Soya Broth, BIOTECH) and incubated at $35^{\circ} \mathrm{c}$ for $4-6$ hours. The inoculum for primary sensitivity testing was prepared from a broth that has been incubated for 4-6 hours. The density of the suspension was adjusted by adding the bacterial suspension to a sterile saline tube to match the density of the desired 0.5 McFarland standards. The surface of Muller-Hinton agar plate was evenly inoculated with the organisms using a sterile swab. The swab was dipped into the suspension and pressed against the side of the test tube to remove excess fluid. The wet swab was then used to inoculate the Muller-Hinton agar, evenly streaked across the surface. By means of Disc Dispenser the antibiotic discs were applied to the surface of the inoculated agar and the plates were incubated overnight at $37^{\circ}$. The diameter of zone of inhibition was measured by using digital caliper and classified as sensitive (S), intermediate (I), or resistance (R). The results were interpreted according to the standard recommendations of the Clinical and Laboratory Standards Institute [12].

\subsection{Data Entry and Analysis}

Response to antibiotics were recorded as either Sensitive (S), Intermediate (I), or Resistant (R) according to the standard recommendations of the Clinical and Laboratory Standards Institute [12]. All the data were entered into the computer and summarized by descriptive statistics of SPSS 20 software. Chi-squared test was used to see the association of variables such as kebeles, rodent species and sex with antimicrobial resistance of the Escherichia coli isolates. P-values were considered statistically significant when $\leq 0.05$.

\subsection{Reference Strains}

Pseudomonus aeruginosa (ATCC-27853), Staphyloccocus aureus (ATCC-25923) and Escherchia coli (ATCC-25922) were used as a quality control throughout the study for culture and antimicrobial resisitance testing. All the strains were obtained from Ethiopian Public Health Institute. 
Antibacterial Resistance of Escherichia coli from Rectal Swabs of Synanthropic Rodents Trapped from Household Compounds in Wolaita Zone, Southern Ethiopia

\section{RESULTS}

\subsection{Distribution of Synanthropic Rodents}

Out of a total of 77 rodents 35 and 42 were females and males, respectively. Nearly equal number of rodents were trapped from both Fate and Abaya Chokare kebeles (Table 1).

Table1. Distribution of small mammals trapped from household compounds in the study area

\begin{tabular}{|l|c|c|c|}
\hline \multirow{2}{*}{ Kebele } & \multicolumn{2}{|c|}{ Rodents } & \multirow{2}{*}{ Total } \\
\cline { 2 - 3 } & Male & Female & \\
\hline Fate & 22 & 16 & 38 \\
\hline Abaya Chokare & 20 & 19 & 39 \\
\hline Total & $\mathbf{4 2}$ & $\mathbf{3 5}$ & $\mathbf{7 7}$ \\
\hline
\end{tabular}

\subsection{Distribution of Synanthropic Rodent Species and Escherichia coli Isolates}

Escherichia coli isolates from all of the rodent species were confirmed biochemically. The rate of biochemically confirmed $E$. coli matched with the number of each rodent species examined (Table 2).

Table2. Rate of occurrence of biochemically confirmed Escherichia coli in synanthropic rodent species examined

\begin{tabular}{|l|l|l|l|l|}
\hline Rodent species & $\begin{array}{l}\text { No. of } \\
\text { Rodennt } \\
\mathrm{s}\end{array}$ & $\%$ & $\begin{array}{l}\text { No. of } \\
\text { E.coli }\end{array}$ & $\begin{array}{l}\text { \% of biochemically } \\
\text { confirmed E.coli }\end{array}$ \\
\hline Stenocephalemys albipes & 24 & 31.2 & 24 & 31.16 \\
\hline Mus mohamet & 18 & 23.4 & 18 & 23.37 \\
\hline Arvicanthis & 15 & 19.5 & 15 & 19.48 \\
\hline Mastomys erythroleucus & 12 & 15.6 & 12 & 15.58 \\
\hline Gerbriliscus species & 4 & 5.2 & 4 & 5.19 \\
\hline Crocidura oliver & 3 & 3.9 & 3 & 3.89 \\
\hline Acomys wilsoni & 1 & 1.3 & 1 & 1.29 \\
\hline Total & $\mathbf{7 7}$ & $\mathbf{1 0 0 \%}$ & $\mathbf{7 7}$ & $\mathbf{1 0 0}$ \\
\hline
\end{tabular}

\subsection{Antimicrobial Resistance Pattern of Escherichia coli Isolates from Synanthropic Rodents}

The Escherichia coli isolates from Arvicanthis was found to be resistant for 7 antimicrobial agents out of the 12 antimicrobial drugs used for susceptibility test. Moreover, E. coli isolates from Crocidura oliver and Mastomys erythroleucus were found to be resistant for 6 antimicobial drugs among the 12 antimicrobial drugs used for susceptibility test. The rest of the isolates from different rodent species had lower resistant rates ranging from 3-5 antimicrobial agents. All E. coli isolates from different rodent species had shown $100 \%$ resistance to amoxacilin and amoxacilin clavulanic acid. On the other hand, all E. coli isolates from different rodent species had shown $100 \%$ resistance to amoxicilin and amoxicilin-clavunalic acid (Table 3).

Table3. Percentage of antibiotic resistance of E.coli isolates from different synanthropic rodents trapped in wolaita Zone, Southern Ethiopia to antimicrobial agents by disc diffusion method

\begin{tabular}{|l|c|c|c|}
\hline $\begin{array}{l}\text { Class and antibiotic } \\
\text { (Abbreviation) }\end{array}$ & Disc content $(\mu \mathrm{g})$ & $\begin{array}{l}\text { Diffusion zone break point }(\mathrm{mm}) \\
\text { (NCCLS break point) }\end{array}$ & $\mathrm{R}(\%)$ \\
\hline $\begin{array}{l}\text { B-Lactam } \\
\text { Ampicillin (Am) }\end{array}$ & 10 & $\leq 13$ & $36(46.7)$ \\
\hline Amoxicillin (Amx) & 30 & $\leq 12$ & $77(100)$ \\
\hline $\begin{array}{l}\text { Amoxicillin-Clavulanic acid } \\
\text { AmC) }\end{array}$ & 30 & $\leq 13$ & $77(100)$ \\
\hline $\begin{array}{l}\text { Cephalosphorins } \\
\text { Ceftazidime (CAZ) }\end{array}$ & 30 & $\leq 17$ & $4(5.19)$ \\
\hline Ceftriaxone (Cro) & 30 & $\leq 19$ & $4(5.19)$ \\
\hline $\begin{array}{l}\text { Aminoglycosides } \\
\text { Gentamycine (Gen) }\end{array}$ & 30 & $\leq 12$ & $0(0.00)$ \\
\hline $\begin{array}{l}\text { Tetracyclines } \\
\text { Tetracycline (TE) }\end{array}$ & 30 & $\leq 11$ & $15(19.48)$ \\
\hline Cloxacillin (Ob) & 5 & $\leq 10$ & $76(98.7)$ \\
\hline
\end{tabular}

International Journal of Research Studies in Biosciences (IJRSB)

Page $\mid 25$ 
Antibacterial Resistance of Escherichia coli from Rectal Swabs of Synanthropic Rodents Trapped from Household Compounds in Wolaita Zone, Southern Ethiopia

\begin{tabular}{|l|c|c|c|}
\hline $\begin{array}{l}\text { Nitrofurans } \\
\text { Nitrofurantoin (F) }\end{array}$ & 100 & $\leq 14$ & $3(3.89)$ \\
\hline Fenicols & & $\leq 12$ & $0(0.00)$ \\
\hline Chloramphenicol (Chl) & 30 & & \\
\hline Quinolones & & $\leq 13$ & $2(2.59)$ \\
\hline Nalidixic acid (NA) & 30 & $\leq 15$ & $0(0.00)$ \\
\hline $\begin{array}{l}\text { Fluoroquinolones } \\
\text { Ciprofloxacin (Cip) }\end{array}$ & 5 & & \\
\hline
\end{tabular}

\subsection{Multiple drug-resistance of Escherichia coli}

About 49 and $51 \%$ of E. coli isolates from synanthropic rodents trapped from Fate and Abaya Chokare kebelles, respectively had multiple drug resistance (Table 4).

Table4. Multiple drug resistance of E. coli isolates from rodents in Fate and Abaya chokare kebelles trapped from December 2015 to March 2016.

\begin{tabular}{|l|l|l|l|l|l|}
\hline \multirow{2}{*}{ Study site (locality) } & \multicolumn{5}{|c|}{ Antibiotic resistance N (\%) } \\
\cline { 2 - 6 } & R0 & R1 & R2 & $\geq$ R3 & \\
\hline Damot Gale (Fate) & 0 & 0 & 0 & $38(49.35)$ & \\
\hline Humbo (Abaya Chokare) & 0 & 0 & 0 & $39(50.64)$ & \\
\hline Total & 0 & 0 & 0 & $77(100)$ & \\
\hline
\end{tabular}

Key: $R O=$ Susceptible to all antibiotics tested; $R 1=$ Resistance to 1 antibiotic; $R 2=$ Resistance to 2 antibiotics; $\geq 3 R=$ Resistance to 3 or more antibiotics

\section{DISCUSSION}

In developing countries like Ethiopia, the sanitary profile of rural household compounds is poor and shared by human and domestic animals. In addition, there are synanthropic rodents that inhabit rural household compounds. These rodents include those that naturally live inside houses such as the black house rat, Rattus rattus, and those that migrate to the human settlement areas as a result of fragmentation or loss of their habitats due to human induced land-use land-cover changes. Synanthropic rodents that share rural house hold compounds are considered to pose public health and veterinary risks [13]. The present study attempted to screen synanthropic rodents for Escherichia coli and testing antimicrobial resistance pattern of $E$. coli isolate for 12 commonly used antimicrobial agents in Ethiopia. The rationale behind testing antimicrobial resistance pattern of $E$. coli isolates for 12 commonly used antimicrobials was to check if synanthropic rodents act as reservoirs of drug resistant $E$. coli in the study area.

Among the 12 antimicrobial agents tested, Escherichia coli isolates from all of the rodents screened were shown complete resistance to amoxicillin and amoxicillin-clavunallic acid. It is important to note that amoxicillin and amoxicillin-clavunallic acid are used as "first" line agents for treatment of bacterial infections in humans. This could lead to very high residue effect in faeces and other wastes of humans which can be passed to the rodents hence leading to acquisition of resistance due to antibiotic selection pressure in resident $E$. coli microflora of the intestines of the rodents [8]. A similar study in Nigeria were shown $57 \%$ of $E$. coli isolates of rodents to be resistant against antimicrobial agents including amoxicillin. In the present study, the finding that ciprofloxacillin and chloramphenicol appeared to be the most potent antibiotics against E. coli (based on the fact that no isolates demonstrated resistance except two intermediate isolates to gentamicin) which is in consistence with previous reports in Nigeria $[14,15]$ (Table 3 ). The same study also were revealed the E. coli to be $100 \%$ sensitive to ciprofloxacillin [16]. All the $77 \mathrm{E}$. coli isolates had shown multiple drug resistance (that is resistance against 3 classes and above antimicrobials) (Table 4). This result was in agreement with the findings elsewhere in Kenya [17].

Among the 77 rodents trapped for screening of $E$. coli belonging to order rodentia and family muridae. The rodents belong to species Stenocephalemys albipes 24(31.2\%), Mus mohamet 18(23.4\%), Arvicanthis 15(19.5\%), Mastomys erythroleucus 12(15\%), Gerbriliscus species 4(5.2\%), Crocidura oliveri 3(3\%) and Acomys wilsoni 1(1.3\%). All of the synanthropic rodents trapped inhabit human modified land escapes including scrub lands, grasslands as well as agricultural lands. The animals are also reported to migrate to the human settlement areas and occure as commensal species in close association to humans [18]. This can implies that the animals can potentially carry wide range 
of potentially zoonotic pathogens including $E$. coli to the human settlement areas. Such rodents can interact with house rats, domestic animals and humans and can play substantial role in the epidemiology of infectious diseases of public health and veterinary importance [13]. However, the possibility of being carriers of other uncultured pathogens, multiple drug resistant $E$. coli strains and molecular basis of resistance acquisition and transmission also cannot be ignored.

Therefore, the study highlights the need for implementation of integrated rodent control of rodents that pose public health risks in the study area. Special emphasis should also be given in the use of public health and veterinary antimicrobial agents in an ecosystem shared by humans, domestic animals and rodents in order to reduce reservoirs of drug resistant pathogens such as E. coli.

\section{CONCLUSIONS AND RECOMMENDATIONS}

This study confirmed that the rodents are carriers of microorganism with drug resistance. Since they live and have close contact with human and pet animals, they may present health risk to humans, pet animals and domestic livestock in the geographical location from where they were trapped. The possibility of the being carriers of other uncultured pathogens, multiple drug resistant E. coli strains and molecular basis of resistance acquisition and transmission also cannot be ignored.

It was found that all the isolates have multi-drug resistance, each isolate resisting three or more drugs. The antimicrobial agents especially amoxicillin, cloxacillin, amoxicillin-clavulanic acid, ampicillin, and to some extent tetracycline are at great risk of being resisted. In this study all amoxacillin and cloxacillin resistant $E$. coli were also resistant to amoxicillin-clavulanic acid. The possible explanation for the occurrence of multi-drug resistant $E$. coli in rodents may be the unrestrictive and uncontrolled use of antibiotics for humans and domestic animals treatment.

Furthermore, such findings have implications for human and veterinary medicine regarding antimicrobial usage and subsequent selection of antimicrobial-resistant organisms.

\section{ACKNOWLEDGEMENT}

The author would like to express deepest gratitude to Mr Yishak Abraham (Asst. Prof.) and $\mathrm{Mr}$ Zewdneh Tomass (Asst. Prof.) for their advices, sharing their knowledge by reading and editing the manuscript. The author extends gratitude to Wolaita Sodo University Teaching and Referral Hospital Laboratory staff members for their technical \& material support and also would like to thank Sodo Christian General Hospital for material support.

\section{ETHICS APPROVAL}

Ethical Guidelines Approved By The Research Ethical Review Committee Of The College Of Natural And Computational Sciences (Wolaita Sodo University) Were Followed For Animal Handling.

\section{REFERENCES}

[1] Crivelli Ximena Blanco, MaríaV. Rumi, Julio C. Carfagnini, Osvaldo Degregorio and Adriana B. Bentancor. Synanthropic rodents as possible reservoirs of shiga toxigenic Escherichia coli strains. Cellular and Infection Microbiology. 2012; (2): 134-135.

[2] Nkogwe Comfort, Juliah Raletobana, Alva Stewart-Johnson, Sharianne Suepaul, and Abiodun Adesiyun. Frequency of Detection of Escherichia coli, salmonella spp., And Campylobacter spp. in the Faeces of Wild Rats (Rattus spp) in Trinidad and Tobago .I. J. Vet. Med. 2011. Article ID 6869237.

[3] Nwiyi P., and Erumaka G. Frequency of detection of salmonella spp. and Esherichia coli in the faeces of house rat in Abia State, Nigeria. Res. Opin. Anim. vet. Sci., 2012; 2(10): 521-524.

[4] Cuong, Nhung N. T., Campbell J, Hoa N. T. , Bryan J. E.,Truc, B. T. Kiet V. N. T., Jombart T., Trung N.V., Hien V.B., Thwaites G., Baker S. , Carrique-Mas J. High Levels of Antimicrobial Resistance among Escherichia coli Isolates from Livestock Farms and Synanthropic Rats and Shrews in the Mekong Delta of Vietnam. J.American. Soci. Microbiol. 2015;(81): 812-820.

[5] Bélanger L, Garenaux A, Harel J. Escherichia coli from animal reservoirs as a potential source of human extraintestinal pathogenic E.coli.FEMS.Immunol Med Microbiol. 2011; (62):1-10.

[6] Smith W. H., Green P., Parsell Z. Vero cell toxin in Escherichia coli and related bacteria: Transfer by phage and conjugation and toxic action in laboratory animals, chicken. J. General Microbiol. 2005; (129):3121-3137. 
[7] Kassa T., Gebre-Selassie S., Asrat D. "Antimicrobial susceptibility patterns of thermotolerant Campylobacter strains isolated from food animals in Ethiopia, Vet. Microbiolo. 2007; (119): 82-87.

[8] Costa PM, Loureiro L, Mates AJF. Transfer of multi-drug resistant bacteria between intermingled ecological niches: The interface between humans, animals and environment. Int. J. Environ. Res. Public Health. 2013; (10):278-294.

[9] Licht T.R., Christensen B.B., Krogfelt K.A., Molin S. Plasmid transfer in the animal intestine and other dynamic bacterial populations: the role of community structure and environment. Microbiol. 1999; (145): 2615- 2622.

[10] Mushtaq-UL-Hassan, Hussain.I., Shehzadi, Shaheen M, Mahmood M.S., A. Rafique and Mahmood-ULHassan M. Occurrence of some zoonotic microorganisms in fecal matter of houserat (Rattus rattus) and house mouse (Mus musculus) trapped from various structures. Pakista Vet.J. 2008; 28(4):171-174.

[11] Glenn I.D. "Determination of sample size," Fact Sheet PEOD-6, a series of the Program Evaluation and Organization Development, Florida Cooperative Extension Service, Institute 2002.

[12] CLSI (Clinical and Laboratory Standards Institute). (2014). Methods for dilution in antimicrobial susceptibility tests for bacteria that grow aerobically. Approved standard M7-A3. Accessed on March 28, 2016.

[13] Lambin Eric. F., Annelise Tran, Sophie O Vanwambeke, Catherine Linard, Valérie Soti. Pathogenic landscapes: Interactions between land, people, disease and their animal hosts. I. J. Health. 2010; (9):54.

[14] Okoli IC. Anti-microbial resistance profiles of E. coli Isolated from free range chickens In urban and rural environments of Imo State, Nigeria. Online J. Health Allied Sci., 2006; (1): 3.

[15] Raji MA, Adekeye JO, Kwaga JKP, Bale JOO, Henton M. Biochemical and serological characterization of Escherichia coli isolated from colibacillosis and dead-in-shell embryos in poultry in Zaria, Nigeria. Nig. Vet. J.2006; 27(2): 33-40.

[16] Wakawa HY.; Franklyne EA. Protective effects of Abrus prectorius leaf extract against carbon tetrachloride-induced liver injury in rats. J.Nat.Scienc.Res. 2015; (5):8.17.

[17] Gakuya F.M, Kyule M.N, Gathura P.B Kariuki S. Antimicrobial susceptibility and plasmid from E.coli isolated from Rats. East Africa Medical Journal. 2001; 78 (10): 521- 522.

[18] IUCN. Red list of threatened species. 2008. e. T16730A6325601. Accessed on May 2016.

Citation: Asfaw Tora \& Yishak Abraham, "Antibacterial Resistance of Escherichia coli from Rectal Swabs of Synanthropic Rodents Trapped from Household Compounds in Wolaita Zone, Southern Ethiopia", International Journal of Research Studies in Biosciences (IJRSB), vol. 7, no. 1, pp. 22-28, 2019. http://dx.doi. org/10.20431/2349-0365. 0701004

Copyright: (C) 2019 Authors. This is an open-access article distributed under the terms of the Creative Commons Attribution License, which permits unrestricted use, distribution, and reproduction in any medium, provided the original author and source are credited. 